\title{
Pelvic gauze packing combined with an external fixator for the treatment of unstable pelvic fractures with a huge retroperitoneal hematoma: a case report
}

\author{
Haibo Yu, Haifeng Huang, Yibing Jin \\ Department of General Surgery, Shengzhou People's Hospital (the First Affiliated Hospital of Zhejiang University Shengzhou Branch), Shaoxing \\ University Shengzhou Branch, Shengzhou, China \\ Correspondence to: Haibo Yu, MS. Department of General Surgery, Shengzhou People's Hospital (the First Affiliated Hospital of Zhejiang University \\ Shengzhou Branch), Shaoxing University Shengzhou Branch, Shengzhou, China. Email: 543618046@qq.com.
}

\begin{abstract}
Pelvic fractures with retroperitoneal hematoma are frequent injuries, and there are multiple treatment options for patients. In this case, a 35-year-old female patient suffered an unstable pelvic fracture due to a car crash, accompanied by the formation of a huge retroperitoneal hematoma. The vital signs of patient were not stable. The patient immediately underwent active anti-shock therapies such as multi-channel infusion, blood transfusion, blood volume supplementation, and blood pressure elevation. However, the vital signs of patient remained unstable, the abdominal circumference increased, hemoglobin decreased rapidly, and the plasma protamine paracoagulation $(3 \mathrm{P})$ test was positive. So, tracheal intubation and mechanical ventilation were performed. Experts from various departments were invited to form an emergency multidisciplinary team (MDT), and exploratory laparotomy was selected. Intraoperative exploration revealed the formation of a huge retroperitoneal hematoma, and there was no bleeding or abdominal organ rupture. So, five large gauzes were developed and packed into the extra peritoneal space to stanch bleeding. Following successful hemostasis, the pelvic external fixator was positioned. Re-surgery was performed 78 hours after surgery to remove all gauzes. At 2.5 months postoperatively, the pelvic external fixator was also removed. Ultimately, the patient achieved good surgical results. Pelvic gauze packing combined with an external fixator is a rapid and effective treatment strategy for critical and huge retroperitoneal hematomas caused by unstable pelvic fractures. To our knowledge, this has not been previously reported.
\end{abstract}

Keywords: Gauze packing; external fixator; retroperitoneal hematoma; pelvic fracture; case report

Submitted Sep 08, 2020. Accepted for publication Nov 03, 2020.

doi: 10.21037/apm-20-1994

View this article at: http://dx.doi.org/10.21037/apm-20-1994

\section{Introduction}

Pelvic fractures are frequent, and mostly occur due to highforce injuries such as falls from heights, traffic accidents, etc. Severe cases are potentially fatal due to hemorrhagic shock (1). The most common injuries associated with pelvic fractures are bleeding and retroperitoneal hematoma (2). For patients with hemodynamically unstable pelvic fractures, early treatment is essential and may affect the prognosis. Moreover, for retroperitoneal hematoma caused by unstable pelvic fractures, the first 3 hours following the injury is the most crucial time (3).
There are numerous treatment options, including hemostasis with interventional embolization and gauze packing, and external pelvic fixation. Arvieux et al. reported that for hemodynamically unstable pelvic fractures, arteriographic embolization should be performed initially, and gauze packing can be considered if this is ineffective (4). Osborn et al. allocated hemodynamically unstable pelvic fractures to the pelvic packing and arteriographic embolization groups (5). Comparison results of preoperative preparations, intraoperative procedures, and postoperative efficacy confirmed that gauze packing 
was generally better than hemostasis with intervention. Ishida et al. reported that multiple methods, including transcatheter arterial embolization, preperitoneal pelvic packing, and external fixation of the pelvis, can be used to comprehensively treat active bleeding caused by pelvic fractures (6).

This article reports a case of pelvic fracture with retroperitoneal hematoma that was treated with pelvic gauze packing combined with an external fixation bracket, and achieved good results.

We present the following article in accordance with the CARE reporting checklist (available at http://dx.doi. org/10.21037/apm-20-1994).

\section{Case presentation}

All procedures performed in studies involving human participants were in accordance with the ethical standards of the research ethics committee of Shengzhou People's Hospital and national research committees and with the Helsinki Declaration (as revised in 2013). Written informed consent was obtained from the patient.

\section{Chief complaints}

A 35 -year-old female patient involved in a car crash suffered from pain in multiple areas for 4 hours. She complained of pelvic, sacral, and chest pain.

\section{History of present illness}

On admission, the patient reported that the pain was persistent but tolerable. Her heart rate was 119 beats/min, blood pressure was $96 / 71 \mathrm{mmHg}$, respiratory rate was 28 breaths/min, and blood oxygen saturation was $93 \%$. Her abdominal examination revealed that the abdomen was soft, presented no tenderness, the abdominal circumference was $84 \mathrm{~cm}$, and hemoglobin was $90 \mathrm{~g} / \mathrm{L}$. The patient immediately underwent active anti-shock therapies including multi-channel infusion, blood transfusion, blood volume supplementation, and blood pressure elevation. However, her vital signs remained unstable, and so tracheal intubation and mechanical ventilation were performed. The patient was then admitted to the intensive care unit.

\section{History of past illness}

The patient did not suffer from any previous underlying medical conditions.

\section{Physical examination}

At the intensive care unit, her heart rate was 147 beats/min, blood pressure was $108 / 68 \mathrm{mmHg}$ (maintained by pressor drugs), respiratory rate was 23 breaths/min, and blood oxygen saturation was $100 \%$ (supported by a ventilator). Her abdomen was still soft, but the abdominal circumference had increased to $89 \mathrm{~cm}$.

\section{Laboratory examinations}

Hematological examinations showed that the patient's hemoglobin had dropped from 90 to $64 \mathrm{~g} / \mathrm{L}$, platelet count was $53 \times 10^{9} / \mathrm{L}$, white blood cell count was $14.68 \times 10^{9} / \mathrm{L}$, and procalcitonin was $7.26 \mathrm{ng} / \mathrm{mL}$. Also, her prothrombin time (PT) was $19.3 \mathrm{~s}$ (PT reference value was $11.5 \mathrm{~s}$ ), activated partial thromboplastin time was $60.8 \mathrm{~s}$, D-dimer was $>20 \mathrm{mg} / \mathrm{L}$, and the $3 \mathrm{P}$ test was positive. Blood gas analysis revealed that lactic acid was $5.3 \mathrm{mmol} / \mathrm{L}$, and in ascites the hemoglobin was $56 \mathrm{~g} / \mathrm{L}$.

\section{Imaging examinations}

The enhanced computed tomography (CT) scans of the chest and abdomen revealed the following: (I) multiple sacral fractures and the formation of a huge anterior sacral hematoma; (II) fractures of the superior and inferior rami of bilateral pubes, and left pubic symphysis surface; (III) massive abdominopelvic effusions; and (IV) bilateral rib fractures and contusion of both lungs.

\section{Preoperative diagnosis}

Based on the history of illness, physical examination, as well as laboratory and imaging data, provisional diagnoses of hemorrhagic shock, pelvic fracture, retroperitoneal hematoma, bilateral rib fractures, and contusion of both lungs were made.

\section{Surgical procedures}

Experts from various departments were invited to form an emergency multidisciplinary team (MDT). It was presumed that it would be difficult to stop bleeding quickly and effectively via interventional embolization due to the huge retroperitoneal hematoma accompanied by 


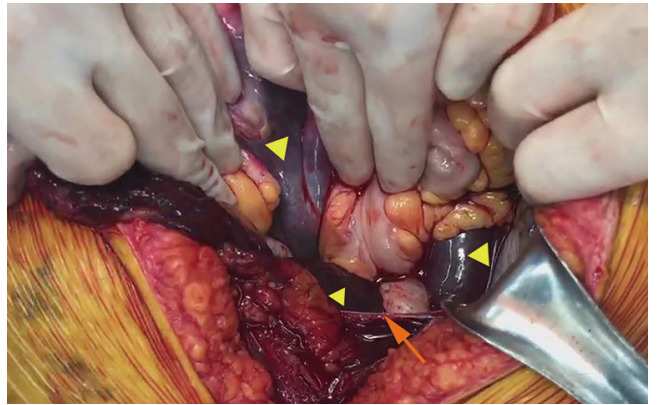

Figure 1 Huge retroperitoneal hematoma (yellow triangle) and peritoneum (orange arrow).

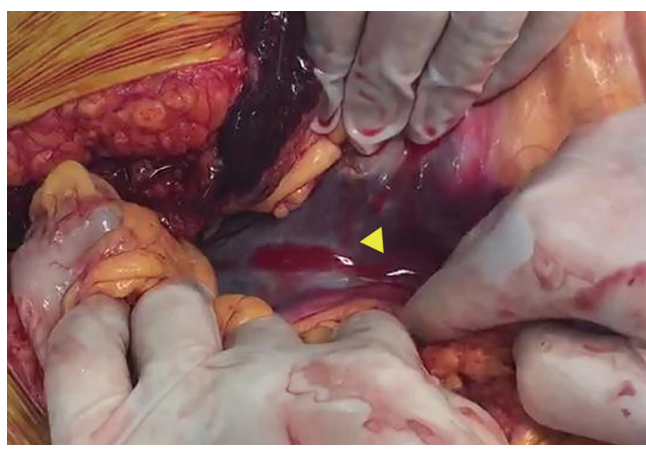

Figure 2 The retroperitoneal hematoma had spread upwards to the root of mesentery (yellow triangle).

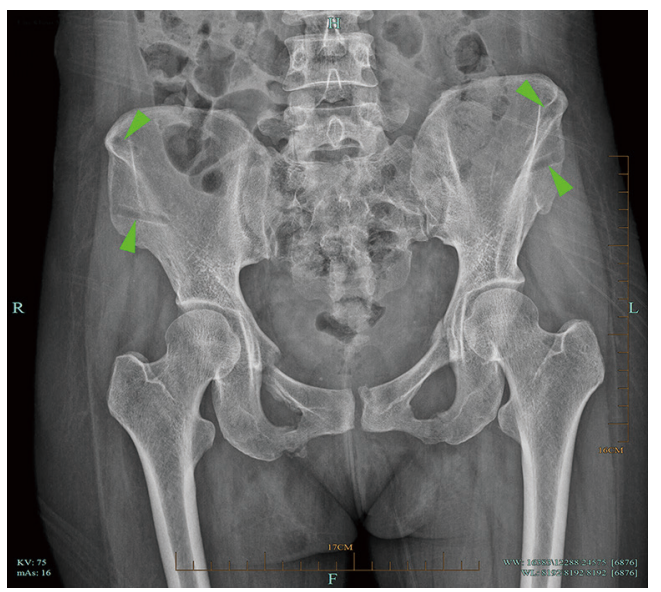

Figure 3 The residual screw channel (green arrow) after removal of the pelvic external fixator.

shock and disseminated intravascular coagulation (DIC). Interpretation of the damage associated with the abdominal cavity was also decided, and exploratory laparotomy was selected as it could explore abdominal organs when treating retroperitoneal hematoma.

After obtaining the consent of patient's family, we immediately sent the patient to the operating room. Intraoperative exploration revealed the formation of a huge retroperitoneal hematoma (Figure 1), which spread upwards to the mesenteric root (Figure 2). No bleeding or abdominal organ rupture were observed, and the peritoneum was intact. A massive abdominopelvic hematocele was observed, and there was no damage to the bladder, urethra, and vagina. The extraperitoneal space was separated, and five large gauzes $(40 \mathrm{~cm} \times 40 \mathrm{~cm})$ were developed and packed into the iliac fossa, presacral space, and retropubic space. $\mathrm{X}$-ray confirmed that the position of the gauze was good. Subsequently, the patient's vital signs gradually stabilized and the vasopressor drugs were discontinued during surgery.

The pelvic external fixator was then positioned. The anterior superior iliac spine and iliac ala on both sides were selected as the screw insertion points. The direction was consistent with that of the chosen pelvic inclination, and a $5 \mathrm{~mm}$ screw was drilled into the iliac bone to a depth of 5-6 cm. The distance of the homolateral screw was approximately $5 \mathrm{~cm}$; the screw was then connected with the connecting rods and fixed to reduce the volume of the pelvis and maintain it constantly.

\section{Outcome and follow-up}

Considering the patient's chest trauma, re-surgery was performed after 78 hours by evaluating the conditions, and then all gauzes were removed. The pelvic external fixator was removed 2.5 months after surgery (Figure 3). After 8 months of follow-up, the patient ultimately achieved good surgical results.

\section{Discussion}

Pelvic fractures are mainly caused by high-force or highspeed injuries, and are often complicated by further organ injuries. The main cause of death is hemorrhagic shock, and the main source of bleeding is from the pelvic venous plexus and the fracture surface (7). Pelvic fractures destroy the structure of the pelvic ring, causing the pelvis to lose its integrity and stability. The volume of the pelvis is increased, which causes the disappearance of the selfpacking effect, and the siphonic and adsorption effects further increase the volume of bleeding. A large amount of blood penetrates into the posterior and lateral peritoneum, 


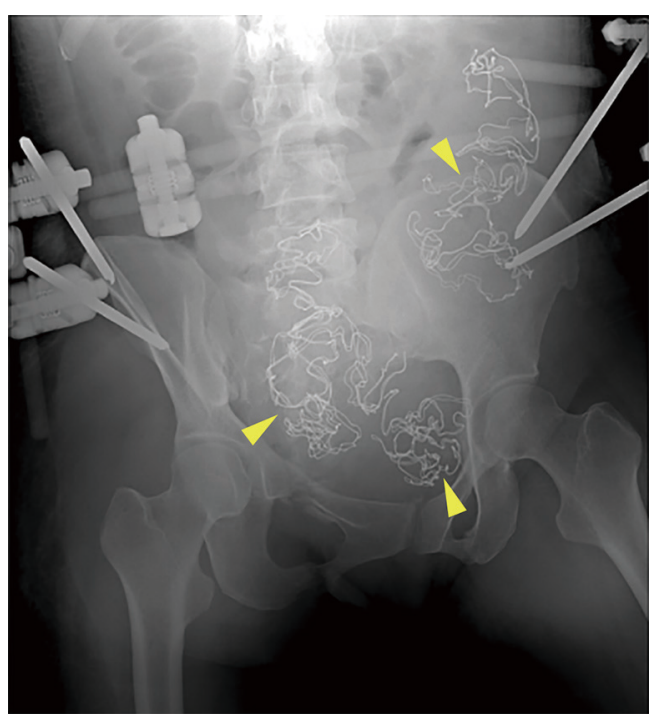

Figure 4 Gauzes were packed into the iliac fossa, presacral space, and retropubic space (yellow arrow).

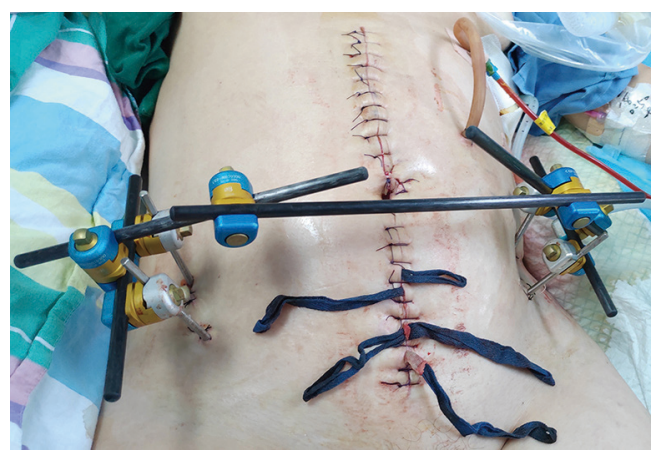

Figure 5 The blue bands of five large gauzes were placed outside the body to facilitate proper removal.

forming a retroperitoneal hematoma, which ultimately leads to hemorrhagic shock. If secondary DIC occurs, it can be considered a life-threatening condition within a short period of time.

The gauze packing technique was first used by Logothetopulos (8) for the treatment of massive hemorrhage caused by open pelvic fracture in 1926. It was then applied in exploratory laparotomy in the 1960s and has since been widely used in clinics. As a part of damage control surgery, pelvic gauze packing is considered to have the following advantages: (I) direct compression of the presacral vascular plexus, with rapid and effective hemostasis, and is especially suitable for treating patients in a critical condition; (II)

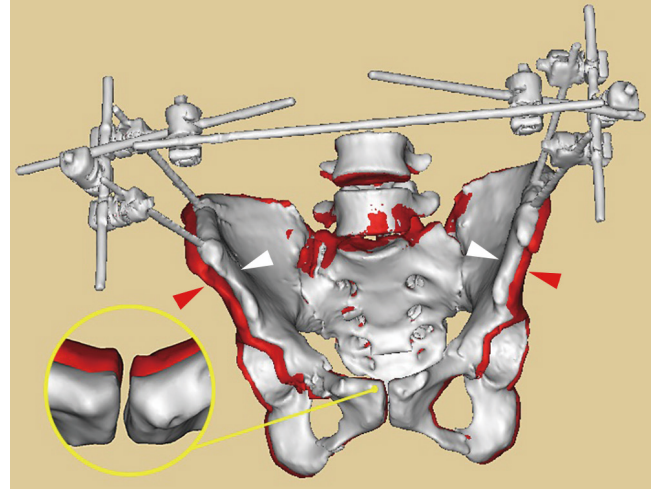

Figure 6 A three-dimensional reconstruction of the pelvis revealed that the volume of the pelvis after external fixation (white arrow) was significantly smaller than before external fixation (red arrow). After external fixation of the pelvis, the position of the pubic symphysis shifts forward, indicating that the pubic symphysis is tight enough, and the pelvic volume has been reduced and remains constant.

rapidly stabilizes vital signs to avoid further DIC and multiorgan dysfunction syndrome, facilitating postoperative recovery; and (III) does not corrode the intestinal canal or cause intestinal adhesions and obstruction, and removal of the gauze is simple and convenient.

During pelvic gauze packing, the following points should be noted: (I) the size of the gauze: larger gauzes should be selected. This is because larger gauzes have a wide compression area, allow for a short operation time, and have a good hemostatic effect. Furthermore, due to the larger size, they are easy for en bloc removal and hard to miss; (II) packing an appropriate number of gauzes according to the extraperitoneal space: excessive packing increases the pressure in the abdominal cavity, leading to abdominal compartment syndrome (9). On the other hand, insufficient packing does not achieve a satisfactory hemostatic effect. Importantly, the number of packed gauzes should be clearly recorded; (III) the position of gauze packing: the gauze is generally packed into the iliac fossa, presacral space, and retropubic space (Figure 4). Improper packing reduces the hemostatic effect, and may even lead to re-bleeding. In this case study, the blue bands of the gauze were placed outside the body after the gauze was packed (Figure 5). This ensured that the gauze would not cause an infection or corrosion upon removal, thereby preventing unnecessary complications.

The pelvic external fixator assists in fixing fractures, 
controlling bleeding, and improving the hemodynamic status, as well as the dual effects of hemostasis and treatment. It offers several advantages including: (I) fixation of the pelvic ring effectively reduces the pelvic volume, provides constant maintenance (Figure 6), and controls bleeding of veins and small arteries, thus preventing rebleeding; (II) reduction and fixation of the fracture reduces exudation at the broken end of the fracture surface; (III) compression of the gauze in the pelvis strengthens and consolidates the hemostatic effect of the retroperitoneal hematoma; and (IV) it is minimally invasive and simple with reliable fixation, which facilitates resuscitation as well as further diagnosis and treatment of associated injuries.

Following fixation of the stent, attention should be paid to the patient's consciousness, breathing, blood pressure, abdominal distension, urine output, etc. (10). They should also be monitored for the occurrence of abdominal compartment syndrome. The external fixator should be checked regularly to avoid complications such as channel infection and screw loosening, and should be generally maintained in a fixed position for 3 months.

Under stable conditions, researchers have recommended removal of the gauze within 48 hours after surgery, and generally no more than 72 hours postoperatively (11). Blood is a good bacterial culture medium, and gauze with a porous structure is conducive to the growth and reproduction of bacteria. So, if gauze is packed for an extended time, secondary infections can easily occur (12). In this case, re-surgery was performed after 78 hours, and the gauzes and blue bands were quickly found and removed. Next, a negative pressure drainage tube was placed into the extraperitoneal space to observe for the presence or absence of re-bleeding, or to discharge effusions of the wound cavity, thereby reducing the risk of infections. This study did not conduct a bacterial culture of the packed gauze during surgery. However, some wound cavity effusions were collected for culturing, and the results showed no bacterial growth.

For treating unstable pelvic fractures with retroperitoneal hematoma, integration of fluid resuscitation, hemostatic resuscitation, and damage control techniques can significantly improve the success rate of treatment (13). This study demonstrated that pelvic gauze packing combined with an external fixator was a rapid and effective treatment strategy for the diagnosis and treatment of critical retroperitoneal hematomas caused by unstable pelvic fractures.

\section{Acknowledgments}

We would like to express special thanks to the Director of Orthopaedics, Hongyu $\mathrm{Xu}$, for the intraoperative guidance of this case.

Funding: None.

\section{Footnote}

Reporting Checklist: The authors have completed the CARE reporting checklist. Available at http://dx.doi.org/10.21037/ apm-20-1994

Conflicts of Interest: All authors have completed the ICMJE uniform disclosure form (available at http://dx.doi. org/10.21037/apm-20-1994). The authors have no conflicts of interest to declare.

Ethical Statement: The authors are accountable for all aspects of the work in ensuring that questions related to the accuracy or integrity of any part of the work are appropriately investigated and resolved. All procedures performed in studies involving human participants were in accordance with the ethical standards of the research ethics committee of Shengzhou People's Hospital and national research committees and with the Helsinki Declaration (as revised in 2013). Written informed consent was obtained from the patient.

Open Access Statement: This is an Open Access article distributed in accordance with the Creative Commons Attribution-NonCommercial-NoDerivs 4.0 International License (CC BY-NC-ND 4.0), which permits the noncommercial replication and distribution of the article with the strict proviso that no changes or edits are made and the original work is properly cited (including links to both the formal publication through the relevant DOI and the license). See: https://creativecommons.org/licenses/by-nc-nd/4.0/.

\section{References}

1. Lee MA, Yu B, Lee J, et al. Effects of the establishment of a trauma center and a new protocol on patients with hemodynamically unstable pelvic fractures at a single institution in Korea. Eur J Trauma Emerg Surg 2019;45:273-9.

2. Cullinane DC, Schiller HJ, Zielinski MD, et al. 
Eastern Association for the Surgery of Trauma practice management guidelines for hemorrhage in pelvic fracture--update and systematic review. J Trauma 2011;71:1850-68

3. Lindahl J, Handolin L, Söderlund T, et al. Angiographic embolization in the treatment of arterial pelvic hemorrhage: evaluation of prognostic mortality-related factors. Eur J Trauma Emerg Surg 2013;39:57-63.

4. Arvieux C, Thony F, Broux C, et al. Current management of severe pelvic and perineal trauma. J Visc Surg 2012;149:e227-38.

5. Osborn PM, Smith WR, Moore EE, et al. Direct retroperitoneal pelvic packing versus pelvic angiography: A comparison of two management protocols for haemodynamically unstable pelvic fractures. Injury 2009;40:54-60.

6. Ishida K, Noborio M, Shimahara Y, et al. Temporal intrailiac balloon occlusion for the treatment of intractable pelvic fracture hemorrhage. Acute Med Surg 2015;3:195-8.

7. Gänsslen A, Hildebrand F, Pohlemann T. Management of hemodynamic unstable patients "in extremis" with

Cite this article as: $\mathrm{Yu} \mathrm{H}$, Huang $\mathrm{H}$, Jin Y. Pelvic gauze packing combined with an external fixator for the treatment of unstable pelvic fractures with a huge retroperitoneal hematoma: a case report. Ann Palliat Med 2020;9(6):4371-4376. doi: 10.21037/ apm-20-1994 pelvic ring fractures. Acta Chir Orthop Traumatol Cech 2012;79:193-202.

8. Logothetopulos K. An absolute safe blood stilling method for vaginal andabdominal gynecological operations.

Zentralbl Gynakol 1926;50:3202-4.

9. Yoong W, Lavina A, Ali A, et al. Abdomino-pelvic packing revisited: An often forgotten technique for managing intractable venous obstetric haemorrhage. Aust N Z J Obstet Gynaecol 2019;59:201-7.

10. Sánchez-Sánchez M, Garcia-de-Lorenzo A, Herrero E, et al. Prevalence of intra-abdominal hypertension (IAH) among patients with severe burns. Burns 2014;40:533.

11. Flint L, Cryer HG. Pelvic fracture: the last 50 years. J Trauma 2010;69:483-8.

12. Granchi TS, Abikhaled JA, Hirshberg A, et al. Patterns of microbiology in intra-abdominal packing for trauma. J Trauma 2004;56:45-51.

13. Singh A, Kumar A, Kumar P, et al. "Beyond saving lives": Current perspectives of interventional radiology in trauma. World J Radiol 2017;9:155-77.

(English Language Editor: A. Kassem) 